\title{
Cultural heritage and new technologies: trends and challenges
}

\author{
Francesco Piccialli $^{1} \cdot$ Angelo Chianese $^{2}$
}

Published online: 24 November 2016

(C) Springer-Verlag London 2016

The relationship between the cultural heritage $(\mathrm{CH})$ domain and new technologies has always been complex and dialectical, often characterized by the pursuit of technologies that can become a "deadweight" during users' cultural experiences.

Innovative applications and services can shorten the distance between cultural spaces, such as museums, art exhibition, historical center and archeological parks, and citizens: Technology can become a facilitator of interactions and connections between all involved actors, and where interventions can be sustainable, where enjoyment can be enhanced and where people can learn more effectively about culture.

Some important challenges still exist, such as supporting users when inside a cultural space, design interoperable and noninvasive ICT solutions, and managing the cultural knowledge.

These challenges are the focus of this theme issue, where international experts all over the world have presented and discussed state-of-the-art solutions and current trends related to the application of new technologies, methodologies and techniques in the cultural heritage domain.

Twelve papers were accepted for this issue.

Francesco Piccialli

francesco.piccialli@unina.it

Angelo Chianese

angelo.chianese@unina.it

1 Department of Mathematics and Applications "Renato Caccioppoli", University of Naples FEDERICO II, Naples, Italy

2 Department of Electrical Engineering and Information Technologies, University of Naples FEDERICO II, Naples, Italy
The paper [1] entitled "Social Recommendation Service for Cultural Heritage" proposes a novel recommender system to individual and group based on artwork features and user experience. Specifically, a novel architecture of recommender system to individual and group based on the features of artwork, context of user and social affinity between users from user experience is illustrated and discussed. The rationale behind the research idea derives from the need to personalize the visitors access to cultural objects, to their knowledge and connections, of a typical cultural space, and to ensure that content and technological containers are effective with respect to its end users.

The paper [2] entitled "Experiencing Touchless Interaction with Augmented Content on Wearable Head Mounted Displays in Cultural Heritage Applications" describes an interactive wearable AR system to augment the environment with cultural information, since the integration of touchless interaction and augmented reality is particularly challenging. The experimental results show the robustness of the proposed method in different scenarios, with various lighting conditions, in terms of the accuracy and sensitivity of the fingertip detection and of the realtime applicability.

The paper [3] entitled "Questions-Matrix Method According to Divided Dimensions of Infographics Evaluation" aims to draw the essential summary according to the important issue of infographic evaluation, for the audience can find the way to improve and create the better infographics, since the visual/image is a very good expression in the cultural heritage domain. To design good infographic, experimental results suggest to focus on the story, tell it nicely, simple and beautiful in good design.

The paper [4] entitled "Using Gamification to Discover Cultural Heritage Locations from Geo-tagged Photos" discusses and analyzes an interesting tool, named 
PhotoTrip, able to autonomously recommend charming, even if not mainstream, cultural heritage locations along travel itineraries. In particular, the proposed solution exploits social networks, crowdsourcing and gamification in order to involve users. After an user evaluation stage for a period of four months, authors claim that common users are effective in helping the system to remove non-relevant photos, even when they have not been previously taught about the meanings of relevant and non-relevant.

The paper [5] entitled "Innovative Technologies for Intangible Cultural Heritage Education and Preservation: the Case of I-Treasures" presents a research study focused on the role of the technology in Intangible Cultural Heritage $(\mathrm{ICH})$ preservation and education. In particular, the authors discuss the experience carried out within an European project named I-Treasures. In details, this research offers an innovative and dynamic evaluation method, where evaluation is not restricted on the static elements of the data collected by questionnaires, but goes beyond and proposes a structural equation methodology, where the performance of the platform is evaluated by the components that constitute the platform and by the relationships that exist between these components.

The paper [6] entitled "Identifying and Ranking Cultural Heritage Resources on Geotagged Social Media for Smart Cultural Tourism Services" presents a twofold research aim: (1) identifying useful cultural heritage resources from geotagged social media and (2) ranking them with respect to the user context. Conducted experiments show that selected a particular geolocation, current social media data combination with ICT, it is possible to find interesting information for tourists, providing dynamic information in favor of any travel agents.

The paper [7] entitled "Tangible Data Souvenirs as a Bridge between a Physical Museum Visit and Online Digital Experience" explores the value of personalized tangible data souvenirs as a bridge between the physical, personal experience of the visit and the digital online experience of staying engaged within a museum environment. The experimental phase has taken into account the $\operatorname{logs}$ and also gathered data documenting the experience of visitors to the exhibition, including interviews and observation. The results show that the uptake of the souvenir was affected by factors outside our control, i.e., the exhibition layout and signage on the exhibit floor.

The paper [8] entitled "Context-Based Infomobility System for Cultural Heritage Recommendation: Tourist Assistant-TAIS" presents an infomobility system named Tourist Assistant (TAIS) for supporting tourists in a region. In particular, the system solves both main tasks related to the infomobility concept: the user actions analysis, the preferences revealing and the cultural heritage recommendation based on the preferences and current situation.
The evaluation phase shows that system finds and ranges the cultural heritage around by the feasible time and can be used for the on-the-fly tourist support during the trip.

The paper [9] entitled "Visualizing Museum Visitors' Behavior: Where Do They Go and What Do They Do There?" presents a framework for collecting and analyzing information about museum visitors' behavior that relies on user-centered design (UCD) process. Authors conducted several interviews with museum curators and personnel in order to understand the requirements of a system that would help them analyze visitor behavior. Initial results from the evaluation are very supportive, suggesting that such a system can be a valuable tool for evaluation of museum exhibit design.

The paper [10] entitled "Confucius Computer-A Philosophical Agent for Intergenerational Philosophical Play with a Computer" presents the design of a system that permits experiencing Confucius teachings virtually and interactively, re-acquainting users with an intangible heritage. The proposed system can measure philosophical intent of the human and generate a meaningful philosophical answer. With glass-box evaluation, the system is able to identify the keywords and topics with an accuracy of 88.72 and $81.20 \%$, respectively. Moreover, an accuracy of $42.59 \%$ was obtained using $k$-NN method. With a blackbox evaluation, more than $70 \%$ of the users gave rating of 4 (agree) or 5 (strongly agree) for the enjoyment and relevance of virtual Confucius's response to their input sentence or question.

The paper [11] entitled "Semantic Infrastructure of a Smart Museum: Towards Making Cultural Heritage Knowledge Usable and Creatable by Visitors and Professionals" presents a theoretical work, which is a mandatory phase of complex system engineering, discussing a smart museum concept based on services with high intelligence level when additional historical sources can be used to semantically enrich the museum collection, including knowledge acquired from visitors and museum professionals. Authors have considered a concept prototype for the selected case study of the History Museum of Petrozavodsk State University, targeting the requirements coming from the museum application domain and with concrete services that such a museum needs.

The paper [12] entitled "Blended Context-Aware Ubiquitous Learning in Museums: Environment, Navigation Support, and System Development" presents a novel learning framework, namely blended context-aware ubiquitous learning (b-learning). To compare the efficacy of this new proposed framework with other learning platforms, a field experiment of the learning activity, "Rocks and Fossils," was conducted in the National Museum of Natural Science, Taiwan. Experimental results demonstrate that the learners had better learning achievements while 
learning in the proposed b-learning environment. Authors suggest that further research is needed to verify the effects of b-learning on learners' motivation.

We would like to appreciate all authors for their valuable contributions to this theme issue.

\section{References}

1. Jung JJ (2016) Social recommendation service for cultural heritage. doi:10.1007/s00779-016-0985-x

2. Gallo L (2016) Experiencing touchless interaction with augmented content on wearable head mounted displays in cultural heritage applications. doi:10.1007/s00779-016-0987-8

3. Vinh P (2016) Question matrix method according to divided dimensions of infographics evaluation. doi:10.1007/s00779-0160988-7

4. Palazzi C (2016) Using gamification to discover cultural heritage locations from geo-tagged photos. doi:10.1007/s00779-016-0989-6

5. Cozzani G (2016) Innovative technologies for intangible cultural heritage education and preservation: the case of i-treasures. doi:10.1007/s00779-016-0991-z
6. Jung JE (2016) Identifying and ranking cultural heritage resources on geotagged social media for smart cultural tourism services. doi:10.1007/s00779-016-0992-y

7. Petrelli D (2016) Tangible data souvenirs as a bridge between a physical museum visit and online digital experience. doi:10.1007/ s00779-016-0993-x

8. Kashevnik A (2016) Context-based infomobility system for cultural heritage recommendation: Tourist Assistant-TAIS. doi:10. 1007/s00779-016-0990-0

9. Lanir J (2016) Visualizing museum visitors' behavior: where do they go and what do they do there? doi:10.1007/s00779-0160994-9

10. Karunanayaka K (2016) Confucius computer: a philosophical agent for intergenerational philosophical play with a computer. doi:10.1007/s00779-016-0995-8

11. Korzun D (2016) Semantic infrastructure of a smart museum: toward making cultural heritage knowledge usable and creatable by visitors and professionals. doi:10.1007/s00779-016-0996-7

12. Tseng J (2016) Blended context-aware ubiquitous learning in museums: environment, navigation support and system development. doi:10.1007/s00779-016-0986-9 